\title{
A transient solution for vesicle electrodeformation and relaxation
}

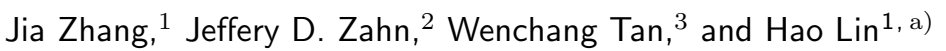 \\ ${ }^{1}$ Department of Mechanical and Aerospace Engineering, Rutgers, The State University of New Jersey, Piscataway, \\ NJ 08854 , USA \\ ${ }^{2}$ Department of Biomedical Engineering, Rutgers, The State University of New Jersey, Piscataway, NJ 08854 , \\ $U S A$ \\ ${ }^{3}$ State Key Laboratory for Turbulence and Complex Systems, Department of Mechanics and Engineering Science, \\ College of Engineering, Peking University, Beijing 100871, China
}

(Dated: 1 November 2018)

\begin{abstract}
A transient analysis for vesicle deformation under DC electric fields is developed. The theory extends from a droplet model, with the additional consideration of a lipid membrane separating two fluids of arbitrary properties. For the latter, both a membrane-charging and a membrane-mechanical model are supplied. The vesicle is assumed to remain spheroidal in shape for all times. The main result is an ODE governing the evolution of the vesicle aspect ratio. The effects of initial membrane tension and pulse length are examined. The model prediction is extensively compared with experimental data, and is shown to accurately capture the system behavior in the regime of no or weak electroporation. More importantly, the comparison reveals that vesicle relaxation obeys a universal behavior regardless of the means of deformation. The process is governed by a single timescale that is a function of the vesicle initial radius, the fluid viscosity, and the initial membrane tension. This universal scaling law can be used to calculate membrane properties from experimental data.
\end{abstract}

\section{INTRODUCTION}

Vesicles are widely used as a model system for biological cells due to their simplicity and controllability. The deformation of the lipid membrane, in particular under an applied electric field (electrodeformation), is often explored to probe membrane properties ${ }^{1,2}$ and to detect pathological changes in cells $\stackrel{3}{*}^{-}$

In the past decade, vesicle electrodeformation has become a significant subject of study, and earlier work can be divided into two categories. In the first category, an alternating-current (AC) field is applied, which often induces stationary and small deformations $\underline{1,2,4,5}$ Correspondingly, an electrohydrodynamic theory in the small-deformation limit was developed to interpret the data trends. $\underline{6}$ In the second, under direct-current (DC) electric fields, vesicles usually exhibit large and transient deformations due to the large field strengths commonly applied $\stackrel{\underline{7}-10}{=}$ Recently, using high-resolution, highspeed optical imaging Riske and Dimova ${ }^{8}$ acquired a large amount of data capturing the complex deformationrelaxation behavior of the vesicles. Although some qualitative and scaling arguments were presented, $\stackrel{4}{\underline{4} \text { the data }}$ was not fully interpreted due to the absence of a predictive model. Meanwhile, one of us (HL) experimentally examined vesicles in the large-deformation regime with aspect ratios reaching ten $\stackrel{10}{-}$ A large-deformation theory was also presented, which provided quantitative agreement with the data therein. However, the model was semi-empirical in that the hydrodynamic problem was not rigorously treated, but followed an empirical approach by Hyuga and co-authors $\stackrel{11,12}{=}$ In general, a rigorous and transient analysis needs to be developed to

\footnotetext{
a) Email address for correspondence: hlin@jove.rutgers.edu
}

understand the complex deformation-relaxation behavior, and to provide insights on the underlying physical processes.

In this work, we develop a transient analysis for vesicle electrodeformation. The theory is derived by extending our previous work on a droplet model,,$\underline{13}$ with the additional consideration of a lipid membrane separating two fluids of arbitrary properties. For the latter, both a membrane-charging and a membrane-mechanical model are supplied. Similar to the droplet model, the main result is also an ordinary differential equation (ODE) governing the evolution of the vesicle aspect ratio. The effects of initial membrane tension and pulse length are examined. The model prediction is extensively compared with experimental data from Riske and Dimova ${ }^{8}$ and Sadik et al. $\stackrel{10}{=}$ and is shown to accurately capture the system behavior in the regime of no or weak electroporation. More importantly, the comparison reveals that vesicle relaxation obeys a universal behavior, and is governed by a single timescale that is a function of the vesicle initial radius, the fluid viscosity, and the initial membrane tension. This behavior is regardless of the means of deformation, either via $\mathrm{AC} / \mathrm{DC}$ electric field, or via mechanical stretching. This universal scaling law is a main contribution of the current work, and can be used to calculate membrane properties from experimental data.

\section{THEORY}

The problem configuration is shown in Fig. 1, Under the influence of an applied electric field, charges of opposite signs are allowed to accumulate on the two sides of the membrane, which induces vesicle deformation and electrohydrodynamic flows both inside and outside the vesicle. We assume that the vesicle remains spheroidal in shape throughout the process. All notations, as well 
as the prolate spheroidal coordinate system follow those from Zhang et al. $\stackrel{13}{n}$ The surface of the prolate spheriod is conveniently given as

$$
\xi=\xi_{0} \equiv \frac{a}{c} .
$$

Here $c=\sqrt{a^{2}-b^{2}}$ is chosen to be the semi-focal length of the spheroidal vesicle, and $a$ and $b$ are the major and minor semi-axis, respectively. For the derivation below, we further assume that the volume of the vesicle is conserved. We subsequently obtain

$$
a=r_{0}\left(1-\xi_{0}^{-2}\right)^{-\frac{1}{3}}, \quad b=r_{0}\left(1-\xi_{0}^{-2}\right)^{\frac{1}{6}} .
$$

Therefore, the vesicle geometry is completely characterized by a single parameter, $\xi_{0}$, which evolves in time along with deformation. The critical idea of the current analysis is to express all variables, e.g., the electric potential and the stream function in terms of $\xi_{0}$. In what follows, we introduce both an electrical and a mechanical model for the membrane. An ODE for $\xi_{0}$ is obtained by applying the stress matching and kinematic conditions.

\section{A. The electrical problem}

The electric potentials both inside and outside the vesicle are described by the Laplace equations:

$$
\nabla^{2} \phi_{i}=\nabla^{2} \phi_{e}=0
$$

However, at the membrane the matching conditions are modified:

$$
\begin{aligned}
\frac{\sigma_{e}}{h_{\xi}} \frac{\partial \phi_{e}}{\partial \xi}=\frac{\sigma_{i}}{h_{\xi}} \frac{\partial \phi_{i}}{\partial \xi}= & C_{m} \frac{\partial \frac{c}{h_{\xi}}\left(\phi_{e}-\phi_{i}\right)}{\partial t} \\
& +\frac{G_{m} c}{h_{\xi}}\left(\phi_{e}-\phi_{i}\right), \quad \text { at } \xi=\xi_{0} .
\end{aligned}
$$

Here $C_{m}$ and $G_{m}$ denote the membrane capacitance and conductance, respectively. $h_{\xi}$ is a metric coefficient of the prolate spheroidal coordinate system. This membranecharging model is commonly adopted by many previous research $\underline{14-18}$ The displacement currents from the electrolytes are not included, which approximation is valid when the Maxwell-Wagner timescale, $T_{M W}=\left(\epsilon_{i}+\right.$ $\left.2 \epsilon_{e}\right) /\left(\sigma_{i}+2 \sigma_{e}\right)$, and the charge relaxation timescale, $T_{c r}=\epsilon / \sigma$, are small when compared with the membranecharging time, $T_{c h}=r_{0} C_{m}\left(1 / \sigma_{i}+1 / 2 \sigma_{e}\right)$, and the deformation time, $T_{d}=\mu_{e} / \epsilon_{e} E_{0}^{2}$. However, the last two times are in general comparable with each other. The first term on the RHS of Eq. (44) represents capacitive charging of the membrane, which includes the effect of membrane deformation. However, the contribution from this effect is usually small, and is neglected in the current analysis for simplicity. Equation (4) can be consequently reduced to

$$
\begin{aligned}
\frac{\sigma_{e}}{h_{\xi}} \frac{\partial \phi_{e}}{\partial \xi}=\frac{\sigma_{i}}{h_{\xi}} \frac{\partial \phi_{i}}{\partial \xi}= & \frac{C_{m} c}{h_{\xi}} \frac{\partial\left(\phi_{e}-\phi_{i}\right)}{\partial t} \\
& +\frac{G_{m} c}{h_{\xi}}\left(\phi_{e}-\phi_{i}\right), \quad \text { at } \xi=\xi_{0} .
\end{aligned}
$$
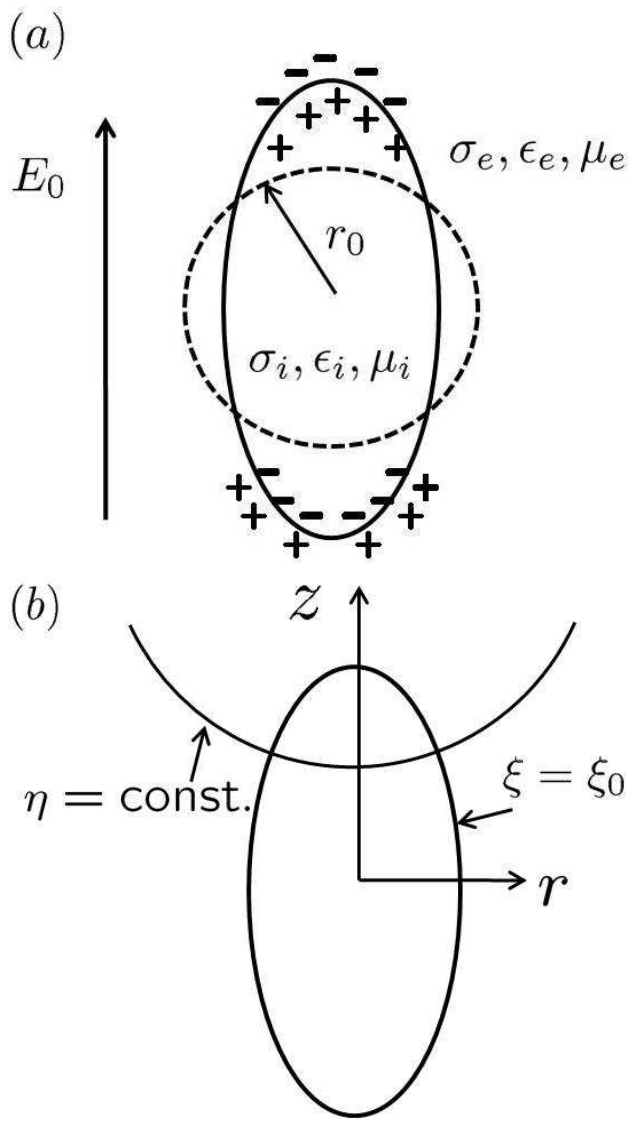

FIG. 1. (a) A schematic of the problem configuration. The original radius of the vesicle is $r_{0}$. The conductivity is denoted by $\sigma$, the permittivity is denoted by $\epsilon$, the viscosity is denoted by $\mu$, and the subscripts $i$ and $e$ denote intravesicular and extravesicular, respectively. The strength of the applied electric field is $E_{0}$. (b) The prolate spheroidal coordinate system.

Equation (5) can be further simplified by considering different stages of charging. In the first stage, the transmembrane potential (TMP), $V_{m} \equiv\left(\phi_{i}-\phi_{e}\right)_{\xi=\xi_{0}}$, grows continuously in magnitude, but the membrane is not permeabilized. Under this condition, $G_{m}$ is near zero, and Eq. (5) becomes

$$
\frac{\sigma_{e}}{h_{\xi}} \frac{\partial \phi_{e}}{\partial \xi}=\frac{\sigma_{i}}{h_{\xi}} \frac{\partial \phi_{i}}{\partial \xi}=\frac{C_{m} c}{h_{\xi}} \frac{\partial\left(\phi_{e}-\phi_{i}\right)}{\partial t}, \quad \text { at } \xi=\xi_{0} .
$$

In the second stage, the maximum TMP reaches the critical threshold, $V_{c}$, for electroporation to occur $\underline{19}-\underline{25}$ The membrane becomes permeable to ions, and $G_{m}$ increases significantly to limit further growth of the TMP. In general, the exact values of $V_{m}$ and $G_{m}$ depend on the detailed electroporation conditions and variables such as pore density and pore area. $\frac{18}{18}$ The solution usually requires a complex numerical simulation which is beyond the scope of the theoretical analysis pursued in this paper. However, a comprehensive model study by $\mathrm{Li}$ and $\operatorname{Lin} \underline{18}$ showed that the maximum TMP remained at the critical level in the presence of the pulse postpermeabilization. In this work, we adopt an approximate 
model for this stage. We assume that once the maximum value of $V_{m}$ reaches $V_{c}$, it no longer grows and "freezes" in time. In addition, the membrane is completely permeabilized, and Eq. (5) is replaced by

$$
\frac{\sigma_{e}}{h_{\xi}} \frac{\partial \phi_{e}}{\partial \xi}=\frac{\sigma_{i}}{h_{\xi}} \frac{\partial \phi_{i}}{\partial \xi}, \quad V_{m}=V_{c}, \quad \text { at } \xi=\xi_{0}
$$

Note that electroporation only occurs for sufficiently strong electric fields, and Eq. (7) is not needed for some of the cases studied below where $V_{c}$ is never reached. Far away from the vesicle surface, the electric field is uniform

$$
-\nabla \phi_{e}=E_{0} \mathbf{z}, \quad \text { at } \xi \rightarrow \infty
$$

We also require that $\phi_{i}$ remains finite at $\xi=1$. For initial condition, we solve Eqs. (3) and (6) with $V_{m}=0$.

The general solution of the electric potentials for both the exterior and interior of the vesicle can be obtained following a similar procedure outlined in Zhang et al.:13

$$
\begin{gathered}
\phi_{e}=E_{0} r_{0}\left[-\lambda \xi+\alpha Q_{1}(\xi)\right] \eta, \\
\phi_{i}=E_{0} r_{0} \beta \xi \eta .
\end{gathered}
$$

Here, $Q_{1}(\xi)$ is a 1st-degree Legendre polynomial of the second kind. $\lambda \equiv c / r_{0}$ is the dimensionless semi-focal length. The coefficients $\alpha$ and $\beta$ are again obtained by applying the matching conditions. In the absence of electroporation, they are given as

$$
\alpha=\frac{\beta+\sigma_{r} \lambda}{Q_{1}^{\prime}\left(\xi_{0}\right) \sigma_{r}}
$$

Here $\sigma_{r} \equiv \sigma_{e} / \sigma_{i}$ is the conductivity ratio. $\tau_{1} \equiv r_{0} C_{m} / \sigma_{i}$ is a membrane-charging time. $\tau_{2} \equiv r_{0} \mu_{e} / \Gamma_{0}$ is a characteristic flow timescale. $\Gamma_{0}$ is the initial membrane tension introduced below. The dimensionless time $\tau$ defined as $\tau \equiv t / \tau_{2}$ has been used. Note that the definition of these times slightly deviates from those used in Zhang et $a l .^{13}$ due to the difference between droplet and vesicle. However, $\tau_{2}$ remains formally the same by replacing $\gamma$ in Zhang et al. 13 with $\Gamma_{0}$.

After the maximum value of $V_{m}$ reaches the critical threshold, electroporation occurs. $\alpha$ and $\beta$ are calculated by Eq. (7) which yields

$\alpha=\frac{-V_{c} /\left(E_{0} r_{0}\right)-\lambda \xi_{0}\left(\sigma_{r}-1\right)}{Q_{1}\left(\xi_{0}\right)-Q_{1}^{\prime}\left(\xi_{0}\right) \xi_{0} \sigma_{r}}, \beta=\left[-\lambda+\alpha Q_{1}^{\prime}\left(\xi_{0}\right)\right] \sigma_{r}$.

The expressions for the normal and tangential electrostatic stresses are found in Zhang et al. 13 and not repeated here.

\section{B. The hydrodynamic problem}

In the regime of low-Reynolds-number flow, the governing equation for the hydrodynamic problem can be rewritten in terms of the stream function, $\psi$, as

$$
\mathrm{E}^{4} \psi=0
$$

Here, the expression for the operator $\mathrm{E}^{2}$ can be found in Dubash and Meste ${ }^{26}$ and Bentenitis and Krause. 27 The stream function is related to the velocity components as

$$
u=-\frac{1}{h_{\xi} h_{\theta}} \frac{\partial \psi}{\partial \xi}, \quad v=\frac{1}{h_{\eta} h_{\theta}} \frac{\partial \psi}{\partial \eta} .
$$

$h_{\eta}$ and $h_{\theta}$ are metric coefficients of the prolate spheroidal coordinate system. At the membrane, $u$ and $v$ represent the tangential and normal velocities, respectively, and they are required to be continuous

$$
u_{e}=u_{i}, \quad v_{e}=v_{i}, \quad \text { at } \xi=\xi_{0} .
$$

In addition, we prescribe a kinematic condition relating the membrane displacement to the normal velocity,

$$
v\left(\xi=\xi_{0}, \eta\right)=\frac{r_{0}\left(1-\xi_{0}^{-2}\right)^{-5 / 6}}{3 \xi_{0}^{2}} \frac{\left(1-3 \eta^{2}\right)}{\sqrt{\xi_{0}^{2}-\eta^{2}}} \frac{d \xi_{0}}{d t} .
$$

At the membrane, the stress matching condition is given as:

$$
\|\tau \cdot \mathbf{n}\|=\mathbf{f}^{m e m}
$$




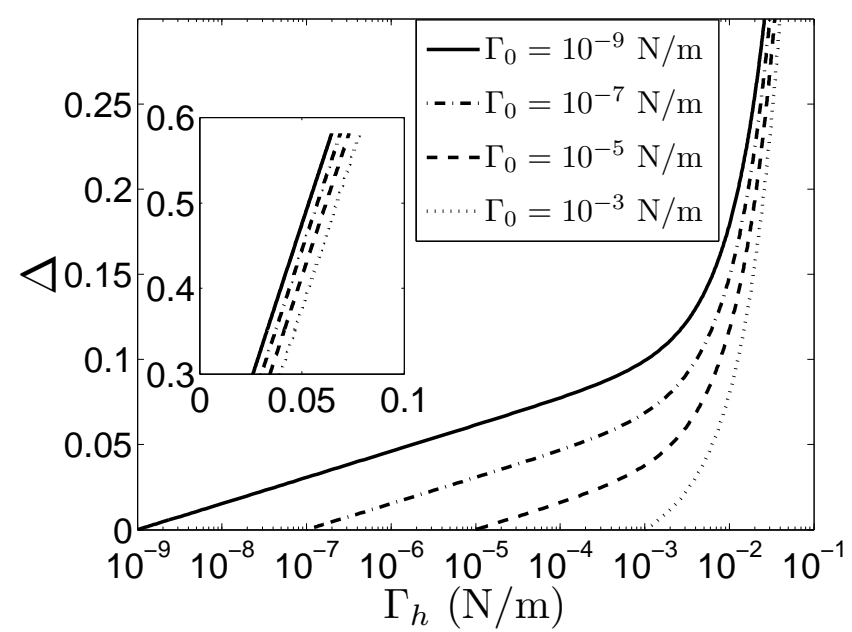

FIG. 2. The relative increase of the apparent area, $\Delta$, as a function of membrane tension, $\Gamma_{h}$, for different values of initial membrane tension, $\Gamma_{0}$. The inset shows the linear regime for larger $\Gamma_{h}$ values.

Here $\mathbf{f}^{m e m}$ is the surface force density arising from the vesicle membrane. The tensor $\tau$ includes contributions from both the hydrodynamic and electrostatic stresses:

$$
\tau \equiv-p \mathbf{I}+\mu\left(\nabla \mathbf{v}+\nabla \mathbf{v}^{T}\right)+\epsilon \mathbf{E E}-\frac{1}{2} \epsilon(\mathbf{E} \cdot \mathbf{E}) \mathrm{I}
$$

\section{The membrane-mechanical model}

The surface force density at the vesicle membrane essentially consists of two parts 6,28

$$
\mathbf{f}^{m e m}=\mathbf{f}^{\kappa}+\mathbf{f}^{\Gamma}
$$

Here $\mathbf{f}^{\kappa}$ is the surface force density induced by bending resistance. $\mathbf{f}^{\Gamma}=2 \Gamma H \mathbf{n}-\nabla_{s} \Gamma$ is the surface force density induced by the membrane tension. $H$ is the mean curvature, and $\Gamma$ is the local membrane tension. We can easily verify that $\mathbf{f}^{\kappa}$ is several orders of magnitude smaller than $\mathbf{f}^{\Gamma}$, and is therefore not included in the current analysis. The local membrane tension, $\Gamma$, is calculated by assuming an effective tension which is uniform over the entire membrane 6.29 An increase of the homogeneous tension, $\Gamma_{h}$, from the initial tension, $\Gamma_{0}$, leads to an increase in the apparent membrane area: ${ }^{1,29}-31$

$$
\Delta=\frac{k_{B} T}{8 \pi \kappa} \ln \frac{\Gamma_{h}}{\Gamma_{0}}+\frac{\Gamma_{h}-\Gamma_{0}}{K_{a}} .
$$

Here $\Delta$ is the increase in the apparent membrane area relative to the initial spherical state,

$\Delta=\frac{1}{2}\left(1-\xi_{0}^{-2}\right)^{-\frac{2}{3}}\left[1-\xi_{0}^{-2}+\left(\xi_{0}^{2}-1\right)^{\frac{1}{2}} \arcsin \left(\xi_{0}^{-1}\right)\right]-1$

$K_{a}$ is the elastic stretching modulus. $\kappa$ is the bending rigidity. Equation (22) indicates that $\Gamma_{0}, \kappa$, and $K_{a}$ are the important parameters in determining membrane tension. $\kappa$ and $K_{a}$ are usually constants for a specific vesicle type, and their values are often readily obtained from previous work ${ }^{1,32,33}$ On the other hand, $\Gamma_{0}$ is specific to an individual vesicle, and its value can not be directly determined from experimental measurements. The relation between $\Delta$ and $\Gamma_{h}$ for different choices of $\Gamma_{0}$ is shown in Fig. 2. When $\Delta$ is small, the membrane area increases through the flattening of the undulations, and $\Gamma_{h}$ shows an exponential correlation with $\Delta$. When $\Delta$ is sufficiently large, a linear behavior is observed instead, and the membrane area increase is mainly due to elastic stretching. Moreover, a larger $\Gamma_{0}$ always leads to a larger $\Gamma_{h}$ for the same value of $\Delta$.

\section{General solution}

A solution for vesicle electrodeformation can be obtained by solving the governing equations of both the electrical and hydrodynamic problems, with the help of the matching conditions. The solution strategy is identical to that presented in Zhang et al., 13 with only differences in the detailed matching conditions for both the electric field and the interfacial forces. For brevity, only the final governing equation for $\xi_{0}$ is presented here:

$$
\begin{gathered}
\frac{d \xi_{0}}{d \tau}=-\frac{1}{F}\left[Q_{N} f_{21}\left(\xi_{0}\right)+Q_{T} \frac{\mu_{r} f_{22}\left(\xi_{0}\right)+f_{23}\left(\xi_{0}\right)}{\mu_{r} f_{14}\left(\xi_{0}\right)+f_{15}\left(\xi_{0}\right)}-\frac{\Gamma_{h}}{\Gamma_{0}} f_{24}\left(\xi_{0}\right)\right] \\
Q_{N}=\frac{C a_{E}}{\lambda^{2}}\left[\left(\lambda-\alpha Q_{1}^{\prime}\left(\xi_{0}\right)\right)^{2}+\left(\lambda-\alpha Q_{1}\left(\xi_{0}\right) / \xi_{0}\right)^{2}-2 \beta^{2} / \epsilon_{r}\right] \\
Q_{T}=\frac{C a_{E}}{\lambda^{2}}\left[\left(\lambda-\alpha Q_{1}^{\prime}\left(\xi_{0}\right)\right)\left(\lambda-\alpha Q_{1}\left(\xi_{0}\right) / \xi_{0}\right)-\beta^{2} / \epsilon_{r}\right] .
\end{gathered}
$$

The functions $f_{14}\left(\xi_{0}\right), f_{15}\left(\xi_{0}\right), f_{21}\left(\xi_{0}\right)-f_{24}\left(\xi_{0}\right)$, and $F$ are the same as those used in Zhang et al. $\frac{13}{2}$ and the detailed expressions are found in the Appendix. $\epsilon_{r} \equiv$ 
$\epsilon_{e} / \epsilon_{i}$ is the permittivity ratio. The factors $Q_{N}$ and $Q_{T}$ again arise from the effects of the tangential and normal stresses, respectively. $C a_{E} \equiv r_{0} \epsilon_{e} E_{0}^{2} / \Gamma_{0}$ is the modified electric capillary number. In the absence of electroporation, the coefficients $\alpha$ and $\beta$ are given in Eqs. (11) and (12). Once the electroporation occurs, Eq. (14) is used instead. Similar to the droplet model, an examination of the three terms in the numerator of Eq. (24a) reveals the contribution from the normal stress, tangential stress, and membrane tension, respectively. The balance between these three terms determines the equilibrium vesicle shape. The above equations are solved until the end of the pulse, $t=t_{p}$.

In the context of vesicle electrodeformation, the relaxation process is equally important, and is more revealing of the underlying physical processes. The governing equations are presented below. In the absence of electro-

poration, Eq. (3) is solved without an applied electric field. The resulting equation for $\xi_{0}$ remains the same as Eq. (24a). The coefficients of $Q_{N}, Q_{T}, \alpha$, and $\beta$ are given as

$$
\begin{gathered}
Q_{N}=\frac{\epsilon_{e} V_{c}^{2}}{\lambda^{2} r_{0} \Gamma_{0}}\left[\alpha^{2}\left(Q_{1}^{\prime 2}\left(\xi_{0}\right)+Q_{1}^{2}\left(\xi_{0}\right) / \xi_{0}^{2}\right)-2 \beta^{2} / \epsilon_{r}\right] \\
Q_{T}=\frac{\epsilon_{e} V_{c}^{2}}{\lambda^{2} r_{0} \Gamma_{0}}\left[\alpha^{2} Q_{1}\left(\xi_{0}\right) Q_{1}^{\prime}\left(\xi_{0}\right) / \xi_{0}-\beta^{2} / \epsilon_{r}\right], \\
\alpha=\frac{\beta}{Q_{1}^{\prime}\left(\xi_{0}\right) \sigma_{r}},
\end{gathered}
$$

$$
\begin{gathered}
{\left[\frac{Q_{1}\left(\xi_{0}\right)}{Q_{1}^{\prime}\left(\xi_{0}\right) \sigma_{r}}-\xi_{0}\right] \frac{d \beta}{d \tau}-\left[\frac{Q_{1}\left(\xi_{0}\right) Q_{1}^{\prime \prime}\left(\xi_{0}\right)-Q_{1}^{\prime 2}\left(\xi_{0}\right)\left(1-\sigma_{r}\right)}{Q_{1}^{\prime 2}\left(\xi_{0}\right) \sigma_{r}} \frac{d \xi_{0}}{d \tau}+\frac{\tau_{2}}{\tau_{1} \lambda}\right] \beta=0,} \\
\alpha\left(\tau_{p}\right)=\frac{V_{m}\left(\tau_{p}\right)}{V_{c}\left(Q_{1}^{\prime}\left(\xi_{0}\right) \xi_{0} \sigma_{r}-Q_{1}\right)}, \quad \beta\left(\tau_{p}\right)=\frac{V_{m}\left(\tau_{p}\right) Q_{1}^{\prime}\left(\xi_{0}\right) \sigma_{r}}{V_{c}\left(Q_{1}^{\prime}\left(\xi_{0}\right) \xi_{0} \sigma_{r}-Q_{1}\right)} .
\end{gathered}
$$

In Eq. (29), the initial conditions for $\alpha$ and $\beta$ are obtained by solving Eqs. (3) and (6), and requiring that $V_{m}$ assumes the value at the end of the pulse. $\tau_{p}$ is the dimensionless time, $t_{p} / \tau_{2}$. Note that in this case, although the pulse is switched off, the electric field is in general not zero, due to the capacitive discharging of the membrane. In this case, the TMP will decreases from its peak value to zero on the membrane-charging timescale, $T_{c h}$.

When electroporation is present, the discharging process is slightly more complex. The full membranecharging model (5) is used. In order to determine the membrane conductance, $G_{m}$, we simply assume that it remains unchanged from the moment the pulse ceases, namely,

$$
G_{m}=-\frac{\sigma_{e} \beta E_{0}}{\lambda V_{c}}
$$

The resulting equation for $\xi_{0}$ again does not formally deviate from Eq. (24a). The coefficients of $Q_{N}, Q_{T}, \alpha$, and $\beta$ are

$$
Q_{N}=\frac{\epsilon_{e} V_{c}^{2}}{\lambda^{2} r_{0} \Gamma_{0}}\left[\alpha^{2}\left(Q_{1}^{\prime 2}\left(\xi_{0}\right)+Q_{1}^{2}\left(\xi_{0}\right) / \xi_{0}^{2}\right)-2 \beta^{2} / \epsilon_{r}\right]
$$

$$
\begin{gathered}
Q_{T}=\frac{\epsilon_{e} V_{c}^{2}}{\lambda^{2} r_{0} \Gamma_{0}}\left[\alpha^{2} Q_{1}\left(\xi_{0}\right) Q_{1}^{\prime}\left(\xi_{0}\right) / \xi_{0}-\beta^{2} / \epsilon_{r}\right], \\
\alpha=\frac{\beta}{Q_{1}^{\prime}\left(\xi_{0}\right) \sigma_{r}},
\end{gathered}
$$

\section{E. A similarity solution for vesicle relaxation}

The governing equation for the relaxation process can be further simplified following two considerations. First, we may ignore the membrane-discharging process. The membrane-charging/discharging time, $T_{c h}$, is on the order of $1 \mathrm{~ms}$, which is in general much shorter than the 
relaxation time observed in the experiments, namely, a few tens of $\mathrm{ms}$ or longer. The relatively small effect of discharging on relaxation is clearly seen in Fig. 3 presented in the following section. Without including the discharging process, the coefficients $Q_{T}$ and $Q_{N}$ in Eq. (24a) are simply set to zero. Second, in the membranemechanical model (22), the first and second term on the RHS represent the effects of undulation unfolding and elastic stretching, respectively. For moderate values of $\Gamma_{0}$, and for small-to-moderate deformations, the second term can be ignored, and the membrane-mechanical model becomes

$$
\Delta=\frac{k_{B} T}{8 \pi \kappa} \ln \frac{\Gamma_{h}}{\Gamma_{0}}
$$

Substituting $Q_{T}=Q_{N}=0$ and Eq. (35) into (24a), we obtain

$$
\frac{d \xi_{0}}{d \tau}=\frac{1}{F} \exp \left(\frac{8 \pi \kappa \Delta}{k_{B} T}\right) f_{24}\left(\xi_{0}\right)
$$

This equation is conveniently rewritten in terms of the aspect ratio as

$$
\frac{d \frac{a}{b}}{d \tau}=-\frac{1}{F} \exp \left(\frac{8 \pi \kappa \Delta}{k_{B} T}\right)\left(\xi_{0}^{2}-1\right)^{-\frac{3}{2}} f_{24}\left(\xi_{0}\right) .
$$

Note that in this equation, $\kappa$, the bending rigidity, is regarded constant for a specific vesicle type, and $\mu_{r}$ (embedded in $F$, see Appendix) is close to 1 as both the fluids are usually aqueous. In addition, $\Delta$, the relative increase of apparent membrane area, depends exclusively on $\xi_{0}$, hence $a / b$ according to Eqs. (23) and (21). Under these assumptions, we observe that Eq. (37) is completely autonomous, and the relaxation process is governed by the dimensionless time, $\tau=t / \tau_{2}$, where $\tau_{2}=r_{0} \mu_{e} / \Gamma_{0}$. This result suggests that the relaxation of vesicles with different initial radius, $r_{0}$, and initial tension, $\Gamma_{0}$, obeys a similarity behavior with the proper scaling suggested above. This behavior is demonstrated by both simulation and analysis of previous experimental data below.

\section{RESULTS}

For all results below, we assume the lipid membrane to be made of egg-PC following Riske and Dimova ${ }^{8}$ (henceforth abbreviated as 'RD05') and Sadik et al. ${ }^{10}$ (henceforth denoted as ' $\mathrm{S} 11^{\prime}$ ). The bending rigidity is taken to be $\kappa=2.47 \times 10^{-20} \mathrm{~J} ;^{1}$ the elastic modulus, $K_{a}=0.14 \mathrm{~N} / \mathrm{m} ; 32,33$ the membrane capacitance, $C_{m}=0.01 \mathrm{~F} / \mathrm{m}^{2} ; 34$ the intravesicular and extravesicular viscosities, $\mu_{i}=\mu_{e}=10^{-3} \mathrm{~Pa} \cdot \mathrm{s}$; the intravesicular and extravesicular permittivities, $\epsilon_{i}=\epsilon_{e}=7 \times 10^{-10} \mathrm{~F} / \mathrm{m}$. The critical transmembrane potential is assumed to be $V_{c}=1 \mathrm{~V}$
TABLE I. List of parameters for Fig. 5. For each case, $E_{0}$ and $t_{p}$ are specified according to RD05. $\Gamma_{0}$ is a fitting parameter to obtain best comparison between simulation and data. For cases b, d, e, and f, extended pulse lengths (denoted by star) are also used.

\begin{tabular}{cccc}
\hline \hline case \# & $E_{0}(\mathrm{kV} / \mathrm{cm})$ & $t_{p}(\mu \mathrm{s})$ & $\Gamma_{0}(\mathrm{~N} / \mathrm{m})$ \\
\hline $\mathrm{a}$ & 1 & 150 & $2.79 \times 10^{-4}$ \\
$\mathrm{~b}$ & 1 & 200 & $3.23 \times 10^{-6}$ \\
& 1 & $300^{*}$ & $3.23 \times 10^{-6}$ \\
$\mathrm{c}$ & 1 & 250 & $1.67 \times 10^{-4}$ \\
$\mathrm{~d}$ & 1 & 300 & $1.80 \times 10^{-6}$ \\
& 1 & $400^{*}$ & $1.80 \times 10^{-6}$ \\
$\mathrm{e}$ & 2 & 50 & $1.80 \times 10^{-4}$ \\
& 2 & $80^{*}$ & $1.80 \times 10^{-4}$ \\
$\mathrm{f}$ & 2 & 100 & $3.16 \times 10^{-6}$ \\
& 2 & $170^{*}$ & $3.16 \times 10^{-6}$ \\
$\mathrm{~g}$ & 3 & 50 & $6.67 \times 10^{-6}$ \\
$\mathrm{~h}$ & 3 & 100 & $3.42 \times 10^{-7}$ \\
\hline \hline
\end{tabular}

\section{A. The effects of $\Gamma_{0}$ and $t_{p}$}

We begin by examining the effects of $\Gamma_{0}$ on vesicle electrodeformation and relaxation. Figure 3 shows the typical system behavior for values of $\Gamma_{0}$ ranging from $10^{-7}-10^{-3} \mathrm{~N} / \mathrm{m}$. The intravesicular and extravesicular conductivities are $\sigma_{i}=6 \times 10^{-4} \mathrm{~S} / \mathrm{m}$ and $\sigma_{e}=4.5 \times 10^{-4} \mathrm{~S} / \mathrm{m}$, respectively following RD05. The field strength is $E_{0}=1 \mathrm{kV} / \mathrm{cm}$, the pulse length is $t_{p}=250 \mu \mathrm{s}$, and the initial radius is $r_{0}=15 \mu \mathrm{m}$. Figure 3(a) shows the evolution of $V_{m}$ at the cathode-facing pole, which demonstrates only a weak dependence on $\Gamma_{0}$. The threshold for electroporation $(1 \mathrm{~V})$ is reached just before the end of the pulse, and its effects are present yet negligible. The discharging occurs on the relatively short timescale of $1 \mathrm{~ms}$ as we discussed above. Figure 3(b) shows the evolution of the aspect ratio, $a / b$. The discharging process manifests itself as a sudden and slight decrease in the aspect ratio immediately after the pulse ceases; its effects can in general be ignored without significantly altering the relaxation behavior. A smaller value of $\Gamma_{0}$ leads to a larger aspect ratio, and a longer relaxation process. The maximum aspect ratio, $[a / b]_{\max }$, is plotted as a function of $\Gamma_{0}$ in Fig. 3(c). As the initial membrane tension decreases toward zero, the maximum achievable aspect ratio saturates.

The similarity behavior in the relaxation process is demonstrated in Fig. 3(d). The descending branches of the curves $\left(t>t_{p}\right)$ shown in Fig. 3(b) are rescaled in terms of $\tau=t / \tau_{2}$, and shifted horizontally. In comparison, the thick solid curve is obtained by directly solving Eq. (37). The convergence of all curves validates that $\tau_{2}=r_{0} \mu_{e} / \Gamma_{0}$ is the single timescale governing vesicle relaxation.

The effects of $t_{p}$ are examined in Fig. 4. The parameters are the same as in Fig. 3, and we fix $\Gamma_{0}$ at $1 \times 10^{-6} \mathrm{~N} / \mathrm{m}$. Figure 4 (a) shows that a longer pulse 

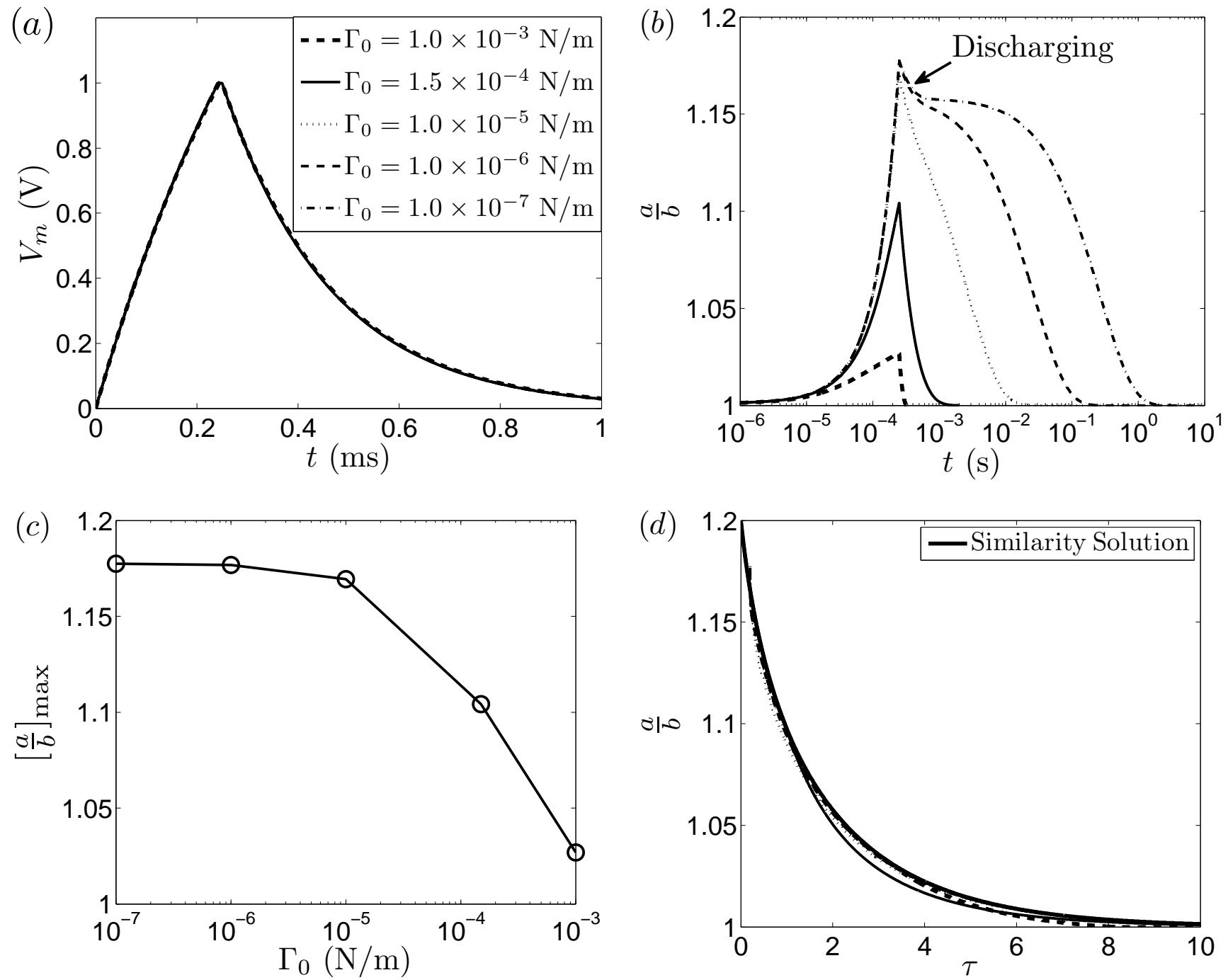

FIG. 3. Vesicle deformation-relaxation as a function of $\Gamma_{0}$. The governing parameters are $\sigma_{i}=6 \times 10^{-4} \mathrm{~S} / \mathrm{m}, \sigma_{e}=4.5 \times 10^{-4} \mathrm{~S} / \mathrm{m}$, $E_{0}=1 \mathrm{kV} / \mathrm{cm}, t_{p}=250 \mu \mathrm{s}$, and $r_{0}=15 \mu \mathrm{m}$. (a) The transmembrane potential at the cathode-facing pole. (b) The time-course of the aspect ratio. (c) The maximum aspect ratio as a function of $\Gamma_{0}$. (d) The similarity behavior in relaxation. The descending branches from (b) are rescaled with $\tau=t / \tau_{2}$. The thick solid curve is directly obtained by integrating Eq. (37).

consistently leads to greater deformation, and the aspect ratio increases along the same envelope. The relaxation times are approximately the same for all cases, because $\tau_{2}$ remains unchanged. The discharging process is in general more conspicuous with longer pulses. In Fig. 4(b), the relaxation curves are again shifted horizontally and rescaled with $\tau_{2}$ to show good agreement with the similarity solution (thick solid line). Note that here because all cases share the same values of $\tau_{2}$, the collapse of the curves is primarily caused by simple shifting. In other words, the aspect ratio also decreases along a common envelope.

The above results are exemplary and demonstrate the typical system behavior. In general, the relaxation process (in particular the relaxation time) is more appreciably affected by the change in $\Gamma_{0}$ than the deformation process. A wide range of pulsing parameters are studied below, in direct comparison with experimental data from RD05 and S11.

\section{B. Comparison with experimental data}

An extensive comparison of our theoretical prediction with the data from RD05 is presented in Fig. 5. For all eight cases, the initial radius is $r_{0}=15 \mu \mathrm{m}$. The electrical conductivities are $\sigma_{i}=6 \times 10^{-4} \mathrm{~S} / \mathrm{m}$ and $\sigma_{e}=4.5 \times 10^{-4} \mathrm{~S} / \mathrm{m}$, respectively, leading to a conductivity ratio of $\sigma_{r}=0.75$. Other parameters are listed in table I. All parameters are taken directly from RD05, except for the extended pulse lengths for some cases noted below. For each case, the initial tension, $\Gamma_{0}$, is deter- 

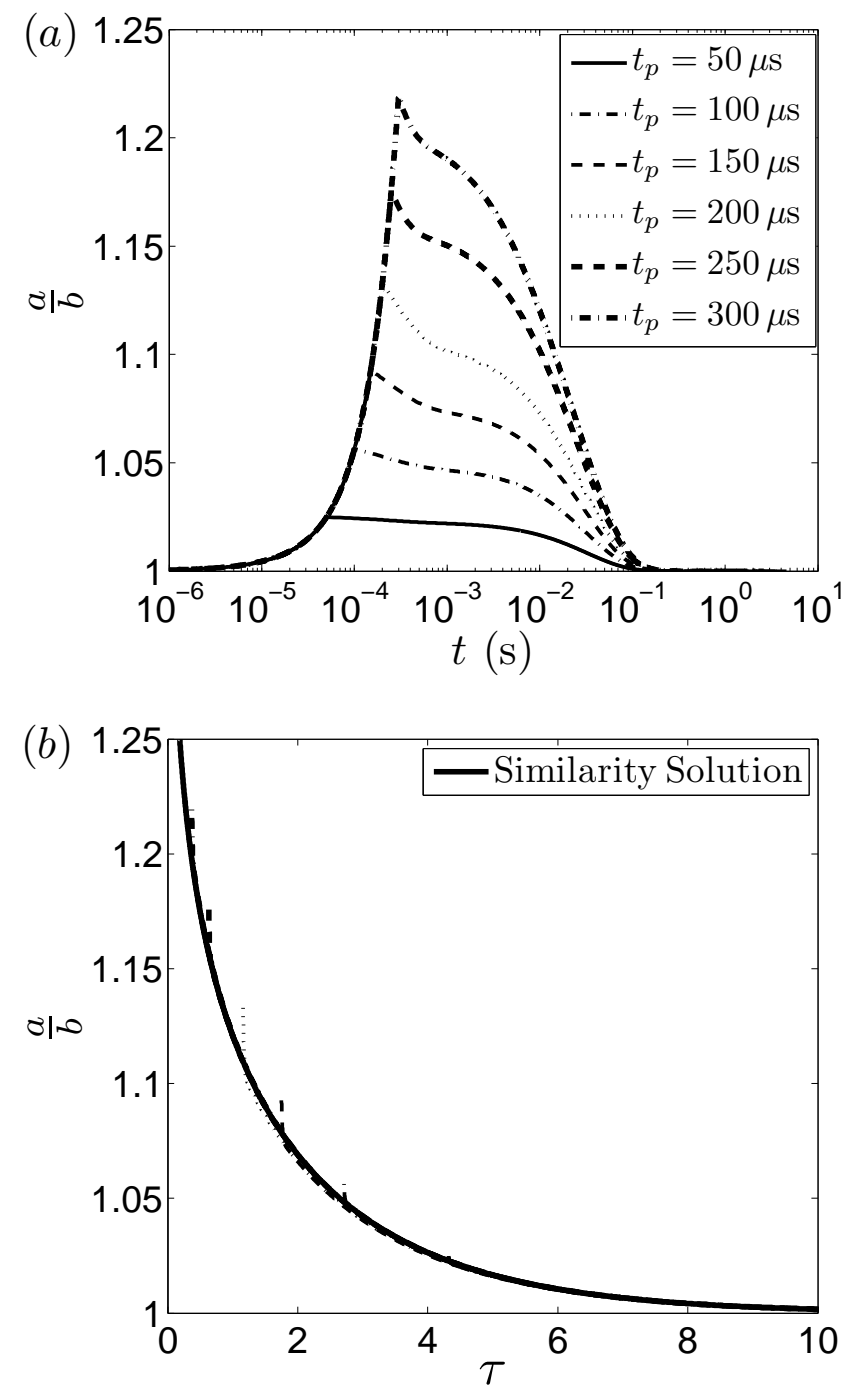

FIG. 4. Vesicle deformation-relaxation as a function of $t_{p}$. The parameters are the same as in Fig. 3 The initial tension is set to be constant, $\Gamma_{0}=1 \times 10^{-6} \mathrm{~N} / \mathrm{m}$. (a) The time-course of the aspect ratio. (b) The similarity behavior is observed by shifting the relaxation curves with respect to time. The relaxation timescale, $\tau_{2}=r_{0} \mu_{e} / \Gamma_{0}$, is the same for all cases. The thick solid curve is directly obtained by integrating Eq. (37).

mined to best fit the experimental data; their values are listed in table I in the last column. The experimental data are presented as symbols; the theoretical predictions, solid lines. In Figs. 5(a) to 5(d), the electric field strength is $E_{0}=1 \mathrm{kV} / \mathrm{cm}$. For these cases, $V_{m}$ is predicted to reach $V_{c}$ at $t=242 \mu \mathrm{s}$. In Figs. 55(a) and 5 (c), good agreements are observed between the theoretical prediction and the data. In Figs. 5(b) and 5(d), the model results underpredict the maximum aspect ratios. This discrepancy is peculiar: our simulation follows the data accurately during the presence of the pulse, which duration is provided by RD05. After the pulse ceases, the simulation predicts immediate relaxation, whereas the vesicles continued to deform in the experiments, due to some unknown cause. In an attempt to mend this difference, we artificially increase the pulse lengths in the simulation in b and d from 200 and 300 to 300 and $400 \mu \mathrm{s}$, respectively. The values for $\Gamma_{0}$ remain unchanged. The results are shown as dashed curves. The model predicts well the data for both the deformation and relaxation processes. Note that although the relaxation curves represented by the solid and dashed lines look somewhat different due to the semi-log scale on the time axis, they actually follow the same descending envelopes which we have demonstrated in Fig. 4(b) above.

In Figs. 5(e) and 5(f), the field strength is increased to be $E_{0}=2 \mathrm{kV} / \mathrm{cm}$, and the pulse lengths used in RD05 were 50 and $100 \mu \mathrm{s}$, respectively. For these cases, our model predicts the occurrence of electroporation around $t=103 \mu \mathrm{s}$. A similar situation is observed as in Figs. 5(b) and 5(d). The solid curves underpredict the maximum aspect ratio. Artificially extending the pulses in e and $\mathrm{f}$ to 80 and $170 \mu \mathrm{s}$, respectively, leads to much better agreement between the two.

In Figs. 5) $(\mathrm{g})$ and 5(h), the field strength is further increased to $3 \mathrm{kV} / \mathrm{cm}$, and electroporation is predicted to occur at $t=66 \mu \mathrm{s}$. The entire deformation-relaxation process is well-captured in $\mathrm{g}$ where $t_{p}=50 \mu \mathrm{s}$. In Fig. 5(h), where $t_{p}=100 \mu \mathrm{s}$, although the model accurately predicts the deformation, the simulated relaxation curve completely deviates from the experimental data. For this case, and for pulses even longer than $100 \mu \mathrm{s}, \mathrm{RD} 05$ [Fig. 1(c) therein] exhibits a regime where complex, multistage relaxation process was observed. In this regime, the membrane structure is likely severely altered due to electroporation, which process can not be captured by our present model. Further comparison with these data is not pursued.

The similarity behavior in the relaxation process is demonstrated in Fig. 6. The experimental data from Figs. 5(a) to 5(g) are shifted horizontally and rescaled with $\tau_{2}$. For each case, $\tau_{2}$ is obtained using $\Gamma_{0}$ listed in table I. The thick solid curve is again the similarity solution from Eq. (37), and the results are shown on both semi-log and linear scales in $\tau$. The coefficient of determination is $R^{2}=0.96$. The experimental data from a wide range of parameters demonstrate a universal behavior governed by a single timescale, $\tau_{2}=r_{0} \mu_{e} / \Gamma_{0}$. This result is a main contribution of the present work.

We remark that a similar behavior should be observed for droplets, where the initial membrane tension, $\Gamma_{0}$, is replaced by $\gamma$, the coefficient of surface tension in $\tau_{2}$ (cf. the definition of $\tau_{2}$ in Zhang et al.) ${ }^{13}$ However, there is a subtle difference between droplet and vesicle relaxation while the coefficient of surface tension is usually a constant, the membrane tension, $\Gamma_{h}$, is not. Nonetheless, as long as $\Gamma_{h}$ depends linearly on $\Gamma_{0}$, which is a good approximation for small-to-moderate deformations. The universal behavior in Fig. [6] is expected.

Finally, the model prediction is compared with data 

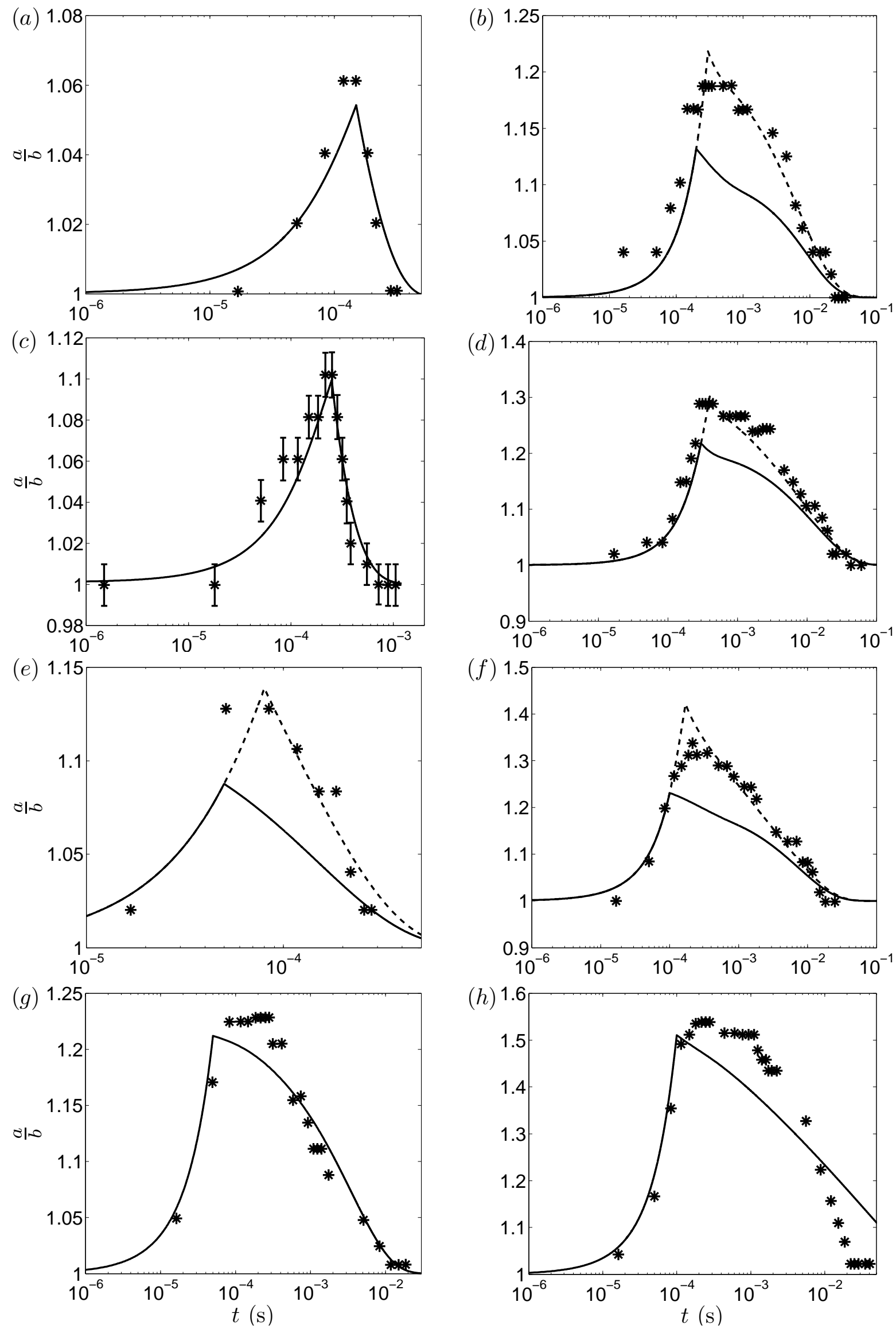

FIG. 5. Comparison with the deformation-relaxation data from RD05. For all cases, $r_{0}=15 \mu \mathrm{m}, \sigma_{i}=6 \times 10^{-4} \mathrm{~S} / \mathrm{m}$, and $\sigma_{e}=4.5 \times 10^{-4} \mathrm{~S} / \mathrm{m}$. Parameters specific to each case are listed in table I The data is represented by symbols, and the simulation is represented by solid curves. For cases b, d, e, and f, the dashed lines represent the simulated results with extended pulses (denoted by stars in table \). 

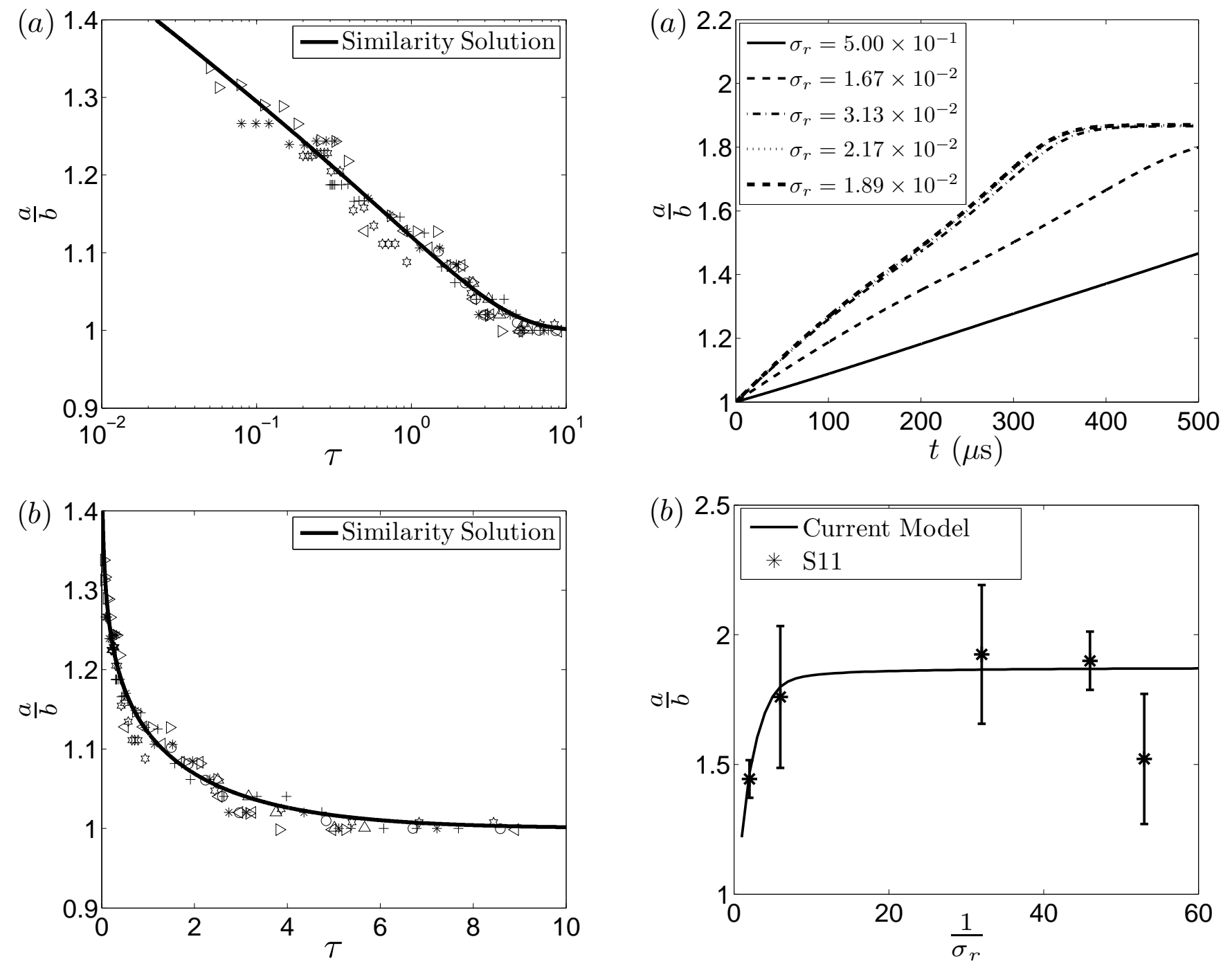

FIG. 6. The similarity behavior of vesicle relaxation. The experimental data from cases a-g in Fig. 5] are shifted in time, then rescaled by $\tau_{2}=r_{0} \mu_{e} / \Gamma_{0}$. They are represented by symbols. The solid curves are calculated with Eq. (37). The same data are shown on both a semi-log (a) and a linear (b) scale. The coefficient of determination is $R^{2}=0.96$.

from S11. In this work, the deformation is examined at a fixed pulse length of $t_{p}=500 \mu \mathrm{s}$, and for five intra-to-extra vesicular conductivity ratios. Only the case of $E_{0}=0.9 \mathrm{kV} / \mathrm{cm}$ is examined, where no or weak electroporation is expected. We do not compare the cases of $E_{0}=2$ and $3 \mathrm{kV} / \mathrm{cm}$ in S11, where the vesicles were in the strongly-electroporated regime, and our model no longer applies. The governing parameters are $r_{0}=11.3 \mu \mathrm{m}$ and $\sigma_{e}=3 \times 10^{-4} \mathrm{~S} / \mathrm{m}$. The initial membrane tension is chosen to be the same for all vesicles, namely, $\Gamma_{0}=1 \times 10^{-8} \mathrm{~N} / \mathrm{m}$. Figure 7 (a) shows the deformation process as a function of time for five conductivity ratios. As $\sigma_{r}$ decreases the rate of deformation increases. Except for the case of $\sigma_{r}=0.5$, the aspect ratio reaches

FIG. 7. Comparison with data from S11. (a) Simulated timecourse of the aspect ratio for various conductivity ratios. For all cases $r_{0}=11.3 \mu \mathrm{m}$ and $\Gamma_{0}=1 \times 10^{-8} \mathrm{~N} / \mathrm{m}$. (b) The aspect ratio at $t=500 \mu \mathrm{s}$ as a function of $1 / \sigma_{r}$.

a plateau before the pulse ends. The time at which the aspect ratio increases saturates with an increasing $\sigma_{r}$. For $\sigma_{r}=0.5$, an equilibrium could be reached if the pulse length is extended and sufficiently long (not shown here). In Fig. $7(\mathrm{~b})$, the aspect ratio at $t=t_{p}$ is shown as a function of $1 / \sigma_{r}$. We choose this representation to facilitate comparison with the data from S11 (symbols), where the definition of the conductivity ratio is $\sigma_{i} / \sigma_{e}$. A reasonable agreement is found between the two. The behavior of the simulation and the data is explained by the dependence of the electrical stress on $\sigma_{r}$ in S11 [see Eq. (21) and Sec. 4 therein]. We do not repeat it here for brevity. The current model represents a significant improvement from that in S11, where the hydrodynamic problem is treated empirically.

Some remarks are appropriate before concluding the 
section. First, for most cases studied here, the TMP is near the threshold, and the vesicles are expected to experience no or weak electroporation. For this regime, our model is shown to provide a good predictive capability, which demonstrates that the membrane-mechanical model (22), although derived assuming no electroporation, can be extended to the weakly-electroporated regime, presumably due to the absence of major structural alterations. Our model is not applicable to the strongly-electroporated regime. Second, the universal scaling law in relaxation observed in Figs. 3, 4, and 6 is expected to hold regardless of the means of deformation, e.g., via $\mathrm{AC} / \mathrm{DC}$ electric fields, or via mechanical stretching. Equation (37) is applicable to a wide range of relaxation phenomena beyond electrodeformation. Third, the current work suggests that an extensive parametric study on vesicle electrodeformation-relaxation experimentally, in particular in the sub-critical regime where electroporation is avoided, can provide the benefit to further validate our model understanding. A systematic approach can be possibly developed based on this work to map membrane properties.

\section{CONCLUSIONS}

In this work, we developed a transient analysis for vesicle electrodeformation. The theory is derived by extending our previous work on a droplet model in Zhang et brane separating two fluids of arbitrary properties. For the latter, both a membrane-charging and a membranemechanical model are supplied. Similar to the droplet model, the main result is also an ODE governing the evolution of the vesicle aspect ratio. The effects of initial membrane tension and pulse length are examined. The initial membrane tension affects the relaxation process much more significantly than the deformation process, in particular when its value is small. The model prediction is extensively compared with experimental data from al., 13 with the additional consideration of a lipid mem-

Riske and Dimova ${ }^{8}$ and Sadik et al., 10 and is shown to accurately capture the system behavior in the regime of no or weak electroporation. More importantly, the comparison reveals that vesicle relaxation obeys a universal behavior, and is governed by a single timescale that is a function of the vesicle initial radius, the fluid viscosity, and the initial membrane tension. This behavior is regardless of the means of deformation, either via $\mathrm{AC} / \mathrm{DC}$ electric field, or via mechanical stretching. This universal scaling law is a main contribution of the current work, and can be used to calculate membrane properties from experimental data.

\section{ACKNOWLEDGMENTS}

JZ and HL acknowledge fund support from an NSF award CBET-0747886 with Dr William Schultz and Dr Henning Winter as contract monitors.

\section{Appendix A}

The functions $f_{14}\left(\xi_{0}\right), f_{15}\left(\xi_{0}\right), f_{21}\left(\xi_{0}\right)-f_{24}\left(\xi_{0}\right)$, and $F$ in Eq. (24a) are given in the following expressions:

$$
\begin{gathered}
f_{11}\left(\xi_{0}\right)=\int \frac{G_{3}(\eta) \eta}{\left(\xi_{0}^{2}-\eta^{2}\right)} d \eta, \\
f_{12}\left(\xi_{0}\right)=\frac{1}{\xi_{0}^{2}-1}\left\{\int \frac{G_{3}(\eta) \eta}{\left(\xi_{0}^{2}-\eta^{2}\right)}\left(\frac{\left(1-3 \eta^{2}\right)}{\left(\xi_{0}^{2}-\eta^{2}\right)}-3\right) d \eta\right\}, \\
f_{13}\left(\xi_{0}\right)=\frac{G_{3}^{\prime \prime}\left(\xi_{0}\right) G_{5}^{\prime}\left(\xi_{0}\right)-G_{3}^{\prime}\left(\xi_{0}\right) G_{5}^{\prime \prime}\left(\xi_{0}\right)}{2 N} \cdot f_{11}\left(\xi_{0}\right),(\mathrm{A} 3) \\
f_{14}\left(\xi_{0}\right)=-\xi_{0} H_{3}^{\prime}\left(\xi_{0}\right) \int \frac{G_{3}(\eta) \eta}{\left(\xi_{0}^{2}-\eta^{2}\right)^{2}} d \eta+\frac{1}{2} H_{3}^{\prime \prime}\left(\xi_{0}\right) f_{11}\left(\xi_{0}\right),
\end{gathered}
$$

$$
f_{15}\left(\xi_{0}\right)=-\frac{H_{3}^{\prime}\left(\xi_{0}\right)\left[G_{3}\left(\xi_{0}\right) G_{5}^{\prime \prime}\left(\xi_{0}\right)-G_{3}^{\prime \prime}\left(\xi_{0}\right) G_{5}\left(\xi_{0}\right)\right]}{2 N} f_{11}\left(\xi_{0}\right)+\xi_{0} H_{3}^{\prime}\left(\xi_{0}\right) \int \frac{G_{3}(\eta) \eta}{\left(\xi_{0}^{2}-\eta^{2}\right)^{2}} d \eta .
$$

Here $N \equiv G_{3}\left(\xi_{0}\right) G_{5}^{\prime}\left(\xi_{0}\right)-G_{3}^{\prime}\left(\xi_{0}\right) G_{5}\left(\xi_{0}\right)$. $G$ and $H$ are Gegenbauer functions of the first and second kind, respectively. The detailed expressions for $G$ and $H$ are found in Dassios et al. ${ }^{36}$

$$
\begin{gathered}
f_{21}\left(\xi_{0}\right)=\frac{1}{2} \xi_{0}^{2} \int \frac{\left(\eta^{2}-1\right)\left(3 \eta^{2}-1\right)}{\left(\xi_{0}^{2}-\eta^{2}\right)} d \eta \\
f_{22}\left(\xi_{0}\right)=\xi_{0} f_{11}\left(\xi_{0}\right)\left[-H_{3}^{\prime}\left(\xi_{0}\right) \int \frac{\left(1-3 \eta^{2}\right)\left(\xi_{0}^{2}-3 \xi_{0}^{2} \eta^{2}+2 \eta^{4}\right)}{\left(\xi_{0}^{2}-\eta^{2}\right)^{2}} d \eta+3 \xi_{0} H_{3}\left(\xi_{0}\right) \int \frac{1-3 \eta^{2}}{\left(\xi_{0}^{2}-\eta^{2}\right)} d \eta\right] \\
f_{23}\left(\xi_{0}\right)=\xi_{0} f_{11}\left(\xi_{0}\right)\left[-\frac{49\left(1-3 \xi_{0}^{2}\right) G_{3}\left(\xi_{0}\right) H_{3}^{\prime}\left(\xi_{0}\right)}{30 N}+H_{3}^{\prime}\left(\xi_{0}\right) \int \frac{\left(1-3 \eta^{2}\right)\left(\xi_{0}^{2}-3 \xi_{0}^{2} \eta^{2}+2 \eta^{4}\right)}{\left(\xi_{0}^{2}-\eta^{2}\right)^{2}} d \eta\right],
\end{gathered}
$$




$$
\begin{gathered}
f_{24}\left(\xi_{0}\right)=\xi_{0}^{3}\left(1-\xi_{0}^{-2}\right)^{\frac{5}{6}} \int \frac{3 \eta^{2}-1}{\left(\xi_{0}^{2}-\eta^{2}\right)^{\frac{3}{2}}} d \eta+\xi_{0}\left(1-\xi_{0}^{-2}\right)^{-\frac{1}{6}} \int \frac{3 \eta^{2}-1}{\sqrt{\xi_{0}^{2}-\eta^{2}}} d \eta \\
F=-\frac{2}{3}\left(f_{25}\left(\xi_{0}\right)+f_{26}\left(\xi_{0}\right) / \mu_{r}\right),
\end{gathered}
$$

where

$$
\begin{aligned}
& f_{25}\left(\xi_{0}\right)=-\frac{f_{22}\left(\xi_{0}\right)}{\xi_{0} f_{11}\left(\xi_{0}\right)} \frac{\left(\mu_{r}-1\right) f_{12}\left(\xi_{0}\right)+f_{13}\left(\xi_{0}\right)}{\mu_{r} f_{14}\left(\xi_{0}\right)+f_{15}\left(\xi_{0}\right)}-3 \xi_{0} \int \frac{3 \eta^{2}-1}{\left(\xi_{0}^{2}-\eta^{2}\right)} d \eta-\frac{\xi_{0}}{\xi_{0}^{2}-1} \int \frac{\left(2 \xi_{0}^{2}-\eta^{2}-1\right)\left(1-3 \eta^{2}\right)^{2}}{\left(\xi_{0}^{2}-\eta^{2}\right)^{2}} d \eta \\
& f_{26}\left(\xi_{0}\right)=-\frac{f_{23}\left(\xi_{0}\right)}{\xi_{0} f_{11}\left(\xi_{0}\right)} \frac{\left(\mu_{r}-1\right) f_{12}\left(\xi_{0}\right)+f_{13}\left(\xi_{0}\right)}{\mu_{r} f_{14}\left(\xi_{0}\right)+f_{15}\left(\xi_{0}\right)}-\frac{49\left(1-3 \xi_{0}^{2}\right) G_{3}^{\prime}\left(\xi_{0}\right)}{30 N}+\frac{\xi_{0}}{\xi_{0}^{2}-1} \int \frac{\left(2 \xi_{0}^{2}-\eta^{2}-1\right)\left(1-3 \eta^{2}\right)^{2}}{\left(\xi_{0}^{2}-\eta^{2}\right)^{2}} d \eta
\end{aligned}
$$

$\mu_{r} \equiv \mu_{e} / \mu_{i}$ is the viscosity ratio.

${ }^{1}$ M. Kummrow and W. Helfrich, "Deformation of giant lipid vesicles by electric fields," Phys. Rev. A 44, 8356-8360 (1991).

${ }^{2}$ G. Niggemann, M. Kummrow, and W. Helfrich, "The bending rigidity of phosphatidylcholine bilayers: Dependences on experimental method, sample cell sealing and temperature," J. Phys. II France 5, 413-425 (1995).

${ }^{3}$ P. K. Wong, W. Tan, and C. M. Ho, "Cell relaxation after electrodeformation: effect of latrunculin a on cytoskeletal actin," Biomech. J. 38, 529-535 (2005).

${ }^{4}$ R. Dimova, K. A. Riske, A. S., N. Bezlyepkina, R. L. Knorr, and R. Lipowsky, "Giant vesicles in electric fields," Soft Matter 3, 817-827 (2007).

${ }^{5}$ S. Aranda, K. A. Riske, R. Lipowsky, and R. Dimova, "Morphological transitions of vesicles induced by alternating electric fields," Biophys. J. 95, L19-L21 (2008).

${ }^{6}$ P. M. Vlahovska, R. S. Gracia, S. Aranda-Espinoza, and R. Dimova, "Electrohydrodynamic model of vesicle deformation in alternating electric fields," Biophys. J. 96, 4789-4803 (2009).

${ }^{7}$ S. Kakorin, T. Liese, and E. Neumann, "Membrane curvature and high-field electroporation of lipid bilayer vesicles," J. Phys. Chem. B 107, 10243-10251 (2003).

${ }^{8} \mathrm{~K}$. A. Riske and R. Dimova, "Electro-deformation and poration of giant vesicles viewed with high temporal resolution," Biophys. J. 88, 1143-1155 (2005).

${ }^{9}$ K. A. Riske and R. Dimova, "Electric pulses induce cylindrical deformations on giant vesicles in salt solutions," Biophys. J. 91, 1778-1786 (2006).

${ }^{10}$ M. M. Sadik, J. Li, J. W. Shan, D. I. Shreiber, and H. Lin, "Vesicle deformation and poration under strong dc electric fields," Phys. Rev. E 83, 066316 (2011).

${ }^{11}$ H. Hyuga, K. J. Kinosita, and N. Wakabayashi, "Deformation of vesicles under the influence of strong electric fields," Jpn. J. Appl. Phys. 30, 1141-1148 (1991).

${ }^{12}$ H. Hyuga, K. J. Kinosita, and N. Wakabayashi, "Deformation of vesicles under the influence of strong electric fields II," Jpn. J. Appl. Phys. 30, 1333-1335 (1991).

${ }^{13}$ J. Zhang, J. D. Zahn, and H. Lin, "A transient solution for droplet deformation under electric fields," arXiv:1210.7878 [physics.flu-dyn] (2012).

${ }^{14}$ H. P. Schwan, "Dielectrophoresis and rotation of cells," in Electroporation and electrofusion in cell biology, edited by E. Neumann, A. E. Sowers, and C. A. Jordan (Plenum Press, 1989).

${ }^{15}$ C. Grosse and H. P. Schwan, "Celluar membrane potentials induced by alternating fields," Biophys. J. 63, 1632-1642 (1992).

${ }^{16}$ K. A. Debruin and W. Krassowska, "Modeling electroporation in a single cell. I. Effects of field strength and rest potential," Biophys. J. 77, 1213-1224 (1999).
${ }^{17}$ W. Krassowska and P. D. Filev, "Modeling electroporation in a single cell," Biophys. J. 92, 404-417 (2007).

${ }^{18} \mathrm{~J}$. Li and H. Lin, "Numerical simulation of molecular uptake via electroporation," Bioelectrochemistry 82, 10-21 (2011).

${ }^{19}$ D. C. Chang and T. S. Reese, "Changes in membrane structure induced by electroporation as revealed by rapid-freezing electron microscopy," Biophys. J. 58, 1-12 (1990).

${ }^{20}$ H. Leontiadou, A. E. Mark, and S. J. Marrink, "Molecular dynamics simulations of hydrophilic pores in lipid bilayers," Biophys. J. 86, 2156-2164 (2004).

${ }^{21}$ A. A. Gurtovenko and I. Vattulainen, "Pore formation coupled to ion transport through lipid membranes as induced by transmembrane ionic charge imbalance: atomistic molecular dynamics study," J. Am. Chem. Soc. 127, 17570-17571 (2005).

${ }^{22}$ M. Tarek, "Membrane electroporation: a molecular dynamics simulation," Biophys. J. 88, 4045-4053 (2005).

${ }^{23} \mathrm{~J}$. Wohlert, W. K. den Otter, O. Edholm, and W. J. Briels, "Free energy of a trans-membrane pore calculated from atomistic molecular dynamics simulations," J. Chem. Phys. 124, 154905 (2006).

${ }^{24}$ U. Pliquett, R. P. Joshi, V. Sridhara, and K. H. Schoenbach, "High electrical field effects on cell membranes," Bioelectrochemistry 70, 275-282 (2007).

${ }^{25}$ M. L. Fernandez, G. Marshall, F. Sagues, and R. Reigada, "Structural and kinetic molecular dynamics study of electroporation in cholesterol-containing bilayers," J. Phys. Chem. B 114, 6855-6865 (2010).

${ }^{26}$ N. Dubash and A. J. Mestel, "Behaviour of a conducting drop in a highly viscous fluid subject to an electric field," J. Fluid Mech. 581, 469-493 (2007).

${ }^{27}$ N. Bentenitis and S. Krause, "Droplet deformation in dc electric fields: the extended leaky dielectric model," Langmuir 21, 61946209 (2005).

${ }^{28}$ U. Seifert, "Configurations of fluid membranes and vesicles," Adv. Phys. 46, 13-137 (1997).

${ }^{29}$ W. Helfrich and R. M. Servuss, "Undulations, steric interaction and cohension of fluid membranes," Il Nuvo Cimento 3D, 137151 (1984).

${ }^{30}$ E. Evans and W. Rawicz, "Entropy-driven tension and bending elasticity in condensed-fluid membranes," Phys. Rev. Lett. 64, 2094-2097 (1990).

${ }^{31}$ E. Evans, "Entropy-driven tension in vesicle membranes and unbinding of adherent vesicles," Langmuir 7, 1900-1908 (1991).

${ }^{32}$ R. Kwok and E. Evans, "Thermoelasticity of large lecithin bilayer vesicles," Biophys. J. 35, 637-652 (1981).

${ }^{33}$ D. Needham, "Cohesion and permeability of lipid bilayer vesicles," in Permeability and Stability of Lipid Bilayers, edited by E. A. Disalvo and S. A. Simon (CRC Press, Boca Raton, FL, 
1995) pp. 49-76.

${ }^{34}$ D. Needham and R. M. Hochmuth, "Electro-mechanical permeabilization of lipid vesicles. Role of membrane tension and compressibility," Biophys. J. 55, 1001-1009 (1989).

${ }^{35} \mathrm{~T}$. Portet and R. Dimova, "A new method for measuring edge tensions and stability of lipid bilayers: effect of membrane com- position," Biophys. J. 99, 3264-3273 (2010)

${ }^{36}$ G. Dassios, M. Hadjinicolaou, and A. C. Payatakes, "Generalized eigenfunctions and complete semiseparable solutions for Stokes flow in spheroidal coordinates," Q. Appl. Math. 52, 157191 (1994). 